\title{
ASO Visual Abstract: Surgical Management of Axilla Following Neoadjuvant Endocrine Therapy
}

\section{Brenna M. Murphy, BS ${ }^{1}$, Tanya L. Hoskin, $\mathrm{MS}^{2}$, Amy C. Degnim, $\mathrm{MD}^{3}$, Judy C. Boughey, $\mathrm{MD}^{3}$, and} Tina J. Hieken, MD ${ }^{3}$

${ }^{1}$ Mayo Clinic Alix School of Medicine, Mayo Clinic, Rochester, MN; ${ }^{2}$ Division of Clinical Trials and Biostatistics, Department of Quantitative Health Sciences, Mayo Clinic, Rochester, MN; ${ }^{3}$ Division of Breast and Melanoma Surgical Oncology, Department of Surgery, Mayo Clinic, Rochester, MN

In our article (https://doi.org/10.1245/s10434-021-1038 5-4), we evaluated the extent and outcomes of axillary surgery in a contemporary cohort of neoadjuvant endocrine therapy (NET) patients, a treatment approach that has become particularly relevant during the COVID-19 pandemic. Complete nodal pathologic response to NET was rare, but de-escalation of axillary surgery among selected

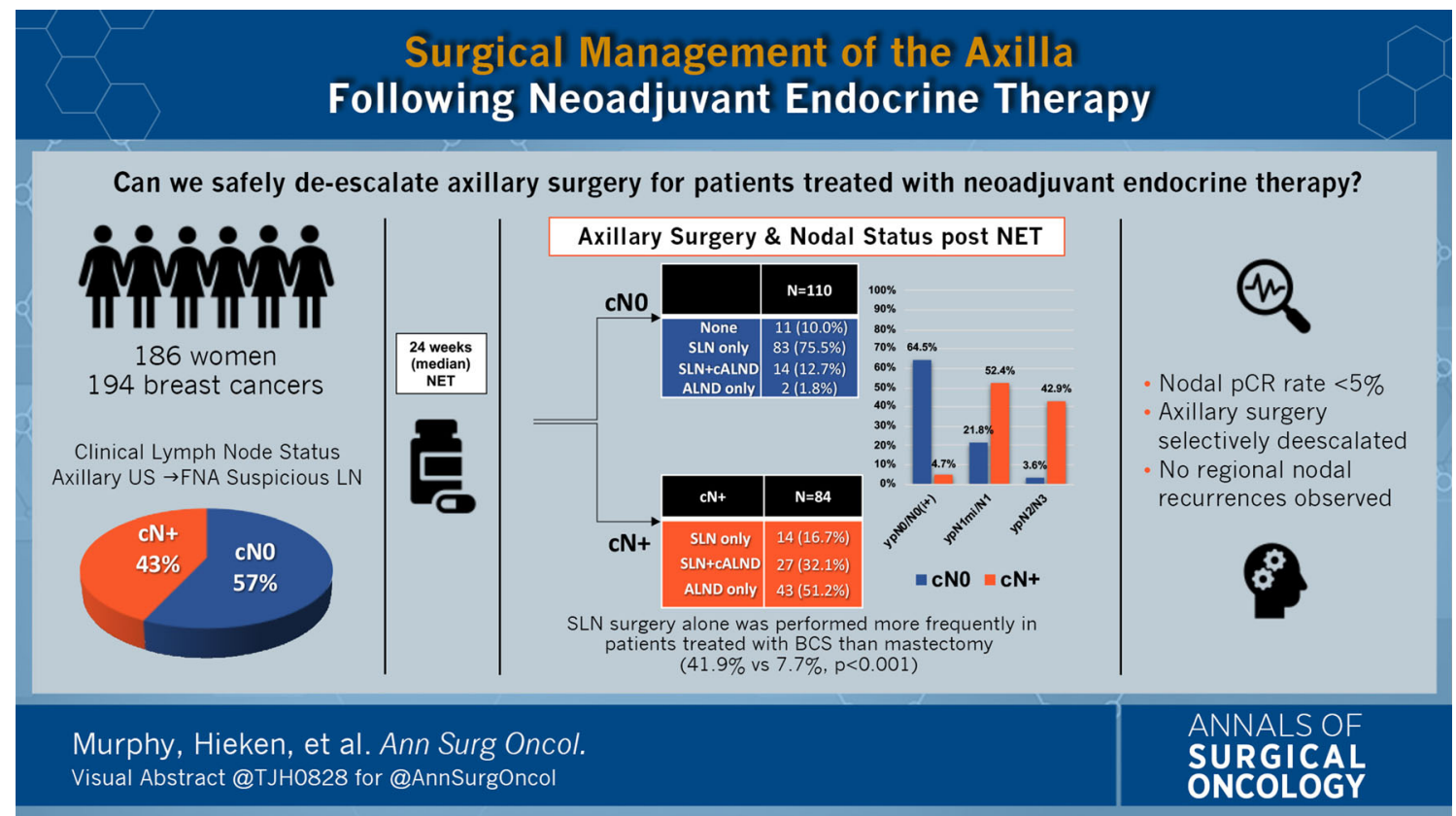

(C) Society of Surgical Oncology 2021

Published Online: 22 July 2021

T. J. Hieken, MD

e-mail: hieken.tina@mayo.edu 
NET patients with positive nodes did not increase regional recurrence rates. Thus, NET patients might be managed similarly to primary surgery patients.

DISCLOSURES None.
Publisher's Note Springer Nature remains neutral with regard to jurisdictional claims in published maps and institutional affiliations. 